\title{
BLOGS PARA PACIENTES COMO NUEVA FUENTE DE INFORMACIÓN DE SALUD
}

Toni González-Pacanowski y Pablo Medina-Aguerrebere
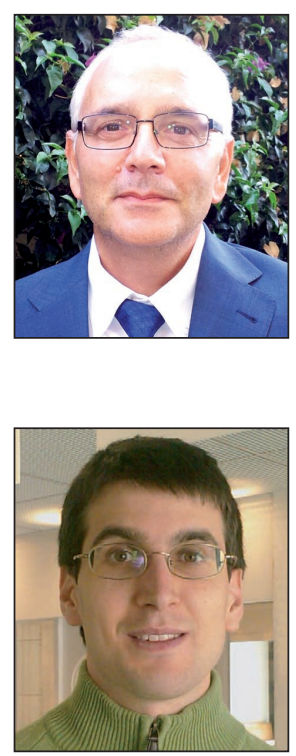

Toni González-Pacanowski es doctor en comunicación por la Universitat Pompeu Fabra (UPF) de Barcelona y profesor de comunicación en la Facultad de Comunicación de la Universidad Internacional de Catalunya. Experto en comunicación interactiva y consultor de nuevas tecnologías en IESE Business School de Barcelona. Colabora con DigiDoc de la UPF y con el Observ. Comunicación y Salud de la Universitat Autònoma de Barcelona. Es co-autor de "Web semántica y sistemas de información documental" (Trea) con un capítulo dedicado a salud y medicina.

Universidad Internacional de Catalunya Edificio Alfa. Campus Barcelona Immaculada, 22. 08017 Barcelona tonigonza@telefonica.net

Pablo Medina-Aguerrebere es doctor en comunicación por la Universidad de Navarra, y profesor de comunicación en la Universidad de Neuchâtel (Suiza). Ha trabajado cuatro años en el sector profesional de la comunicación de salud, y desde el año 2008 realiza labores de investigación académica sobre este tema. Ha publicado "Gestión de la comunicación interna en hospitales" (Fragua) y coordinado junto con Toni González-Pacanowski “Comunicación de la salud: una aproximación multidisciplinar" (Fragua).

Université de Neuchâtel. Faculté des Sciences Économiques Avenue du 1er-Mars 26. CH-2000 Neuchâtel (Suisse) pablomedina@hotmail.fr

\section{Resumen}

Los pacientes recurren cada vez más a los blogs para aprender sobre salud y compartir experiencias relacionadas con esta materia. Son redactados por pacientes o por profesionales de la salud y constituyen una ayuda excepcional para la promoción de la educación de la población en temas de salud. El alto nivel educativo de los bloggers, la variedad de fuentes de información que utilizan y los distintos fines que persiguen convierten a los blogs en actores sanitarios de pleno derecho. Se concluye que el blog constituye un nuevo punto de encuentro entre médico y paciente que influye positivamente en la mejora del servicio de salud ofrecido por los hospitales.

\section{Palabras clave}

Web 2.0, Blogs, Salud, Pacientes, Profesionales de la salud, Socialización.

\section{Title: Blogs as a new source of health information for patients}

\begin{abstract}
Patients are turning increasingly to blogs to learn about health and to share experiences related to this area. These blogs are written by patients or health professionals and constitute an exceptional aid for the promotion of education in health issues for this population. The high educational level of the bloggers, the variety of sources of information used and the different aims pursued make these blogs full participants in the health area. The article concludes that the blog is a new meeting point between doctor and patient that has a positive impact on the improvement of the health service offered by hospitals.
\end{abstract}

\section{Keywords}

Web 2.0, Blogs, Health, Patients, Health professionals, Socialization.

González-Pacanowski, Toni; Medina-Aguerrebere, Pablo. "Blogs para pacientes como nueva fuente de información de salud”. El profesional de la información, 2011, noviembre-diciembre, v. 20, n. 6, pp. 652-658.

http://dx.doi.org/10.3145/epi.2011.nov.08

\section{Introducción}

Internet se ha convertido en una fuente de información sanitaria crecientemente solicitada por los pacientes, que la utilizan para satisfacer sus necesidades informativas y para divulgar y compartir sus experiencias de salud. El rol cada vez más activo de los usuarios y los profesionales de la sanidad, el interés generado por la salud y las facilidades 
ofrecidas por las aplicaciones 2.0 han dado lugar a la creación de blogs de asistencia a pacientes. Los crean usuarios o profesionales de la salud entre otros autores, y representan un desafío comunicativo para las organizaciones sanitarias ya que pueden significar cambios en la relación médico-paciente.

Internet es un proveedor de información de salud para miles de personas

\section{El impacto comunicativo de los blogs de salud}

La aplicación de las nuevas tecnologías de la información y de la comunicación en el sector de la salud ha dado lugar al concepto de medicina 2.0, que se compone, según Eysenbach (2008), de cinco elementos: red social, participación, apomediation (recomendación de información por parte de gente que conoce bien la disciplina tratada), colaboración y apertura. La implantación de la medicina 2.0 encuentra un gran aliado en el software libre, cuya aplicación en el entorno clínico comporta varias ventajas (Rius; González, 2011, p. 205): evita la dependencia del propietario, estimula la innovación tecnológica, facilita la conectividad entre centros, y rebaja costes.

Entre las nuevas aplicaciones de que disponen las empresas del sector sanitario, internet se ha convertido en la más determinante por los beneficios que aporta. Como ya indicaba Parrada (2000, p. 31) hace diez años, la creación de páginas web dedicadas a la salud ofrece varias ventajas para el paciente, como la posibilidad de tener acceso a información sobre enfermedades y sobre tratamientos médicos redactada en un lenguaje universal, lo cual puede favorecer la divulgación médica y la educación sanitaria de la población.

En Estados Unidos la audiencia de las webs sobre salud aumentó un $21 \%$ durante 2007 , mientras que la de webs de información general lo hizo sólo un $5 \%$ (Comscore, 2008). En España un dato ilustra la importancia de los temas de sanidad en internet: en 2009 el 73,9\% de los usuarios utilizó la Red para buscar información de salud, siendo los aspectos más demandados las enfermedades, la nutrición y los medicamentos (Fundación Telefónica, 2010). Sin embargo no toda la información sanitaria disponible en internet es fiable, y aunque lo sea el paciente puede no comprenderla, por lo que no siempre se informa de manera adecuada.

La evolución de internet en los últimos años ha puesto en evidencia su importancia como agente de socialización de la organización y de sus clientes. Los jóvenes españoles de 12 a 18 años utilizan internet en la medida en que les ayuda a su socialización y al aprovechamiento del ocio (Sánchez-Navarro; Aranda, 2011). En este contexto de socialización y de interacción, diferentes organizaciones del sector de la salud han apostado por la implantación de utilidades de la web 2.0. Estas iniciativas resaltan el valor otorgado a las personas que generan contenido en la Red, a la producción colectiva de conocimientos y a la difusión de experiencias personales (Adams, 2010b). La tecnología 2.0 tiene una aplicación práctica en la salud ya que brinda al ciudadano nuevas formas para almacenar, ver, manipular, compartir y experimentar sus datos personales (Bennett; Glasgow, 2009, p. 282), y puede resultar muy beneficiosa para la educación de salud, particularmente con grupos desfavorecidos como discapacitados, personas mayores o residentes en zonas geográficas aisladas (Kamel-Boulos; Wheeler, 2007, p. 17).

Gracias a la web 2.0 el sistema sanitario gira alrededor del paciente y no del hospital

Estas aplicaciones ayudan a que el paciente sea el verdadero protagonista de las acciones de comunicación emprendidas por las empresas pertenecientes al sector de la salud. Ello es positivo, pero implica un riesgo ya que el usuario en ocasiones no posee los conocimientos científicos necesarios para gestionar la información médica de modo fiable. Esta actitud activa del paciente representa un cambio en las relaciones comunicativas que mantiene con el profesional de la salud. En ciertos casos los usuarios que dudan de la opinión del médico acuden a internet para buscar otra que les convenga más y pueden llegar a adoptar dicha información en vez de la facilitada por el profesional sanitario, lo que supone una verdadera amenaza a la confianza entre ambos (Fostier, 2005). Un estudio de Pfizer (2010) demuestra que el $48,5 \%$ de los médicos consideran que muchos de los problemas y conflictos que tienen con los pacientes son debidos a la información que estos consultan en la Red.

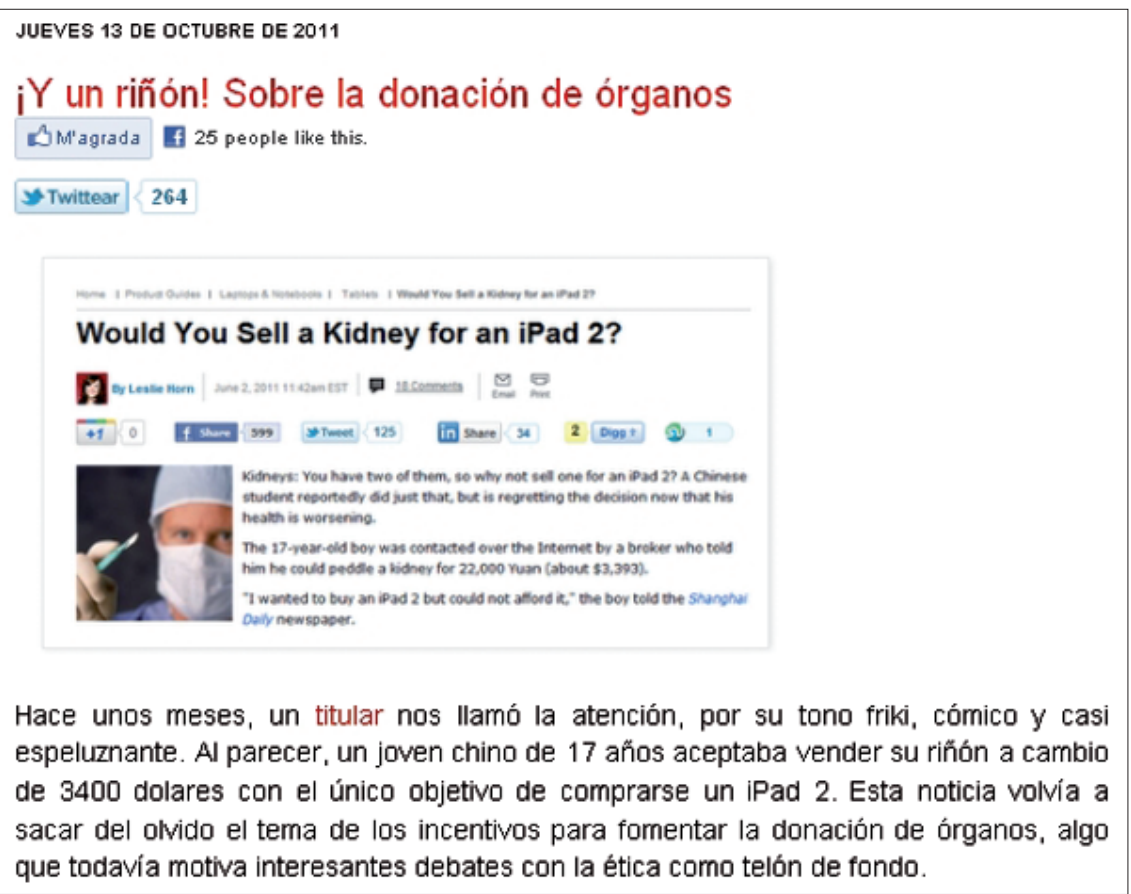

Salud con cosas, http://saludconcosas.blogspot.com 
Entre las aplicaciones de la web 2.0 merece especial atención el blog, que según Orihuela (2006), ayuda a las organizaciones a tener un contacto más directo y personal con sus clientes. En el contexto sanitario existen blogs de profesionales que escriben sobre su práctica diaria y sobre áreas relativas a la medicina, y blogs de pacientes que difunden información sobre ámbitos relativos a la salud y a sus experiencias médicas vividas (Denecke, 2009). El protagonismo adquirido por profesionales y usuarios de la sanidad, sitúa en un lugar secundario a las organizaciones de salud frente al lugar destacado de las personas.

Los blogs redactados por profesionales que tienen experiencia médica y aca-

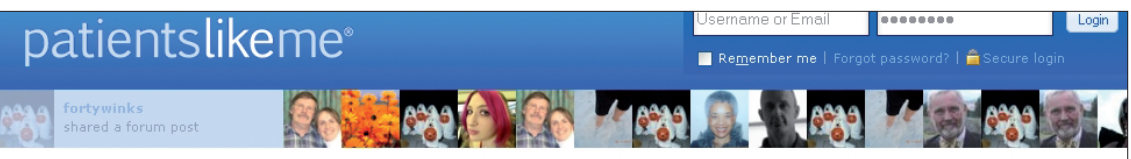

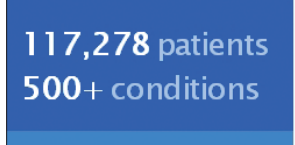

Who's like you?
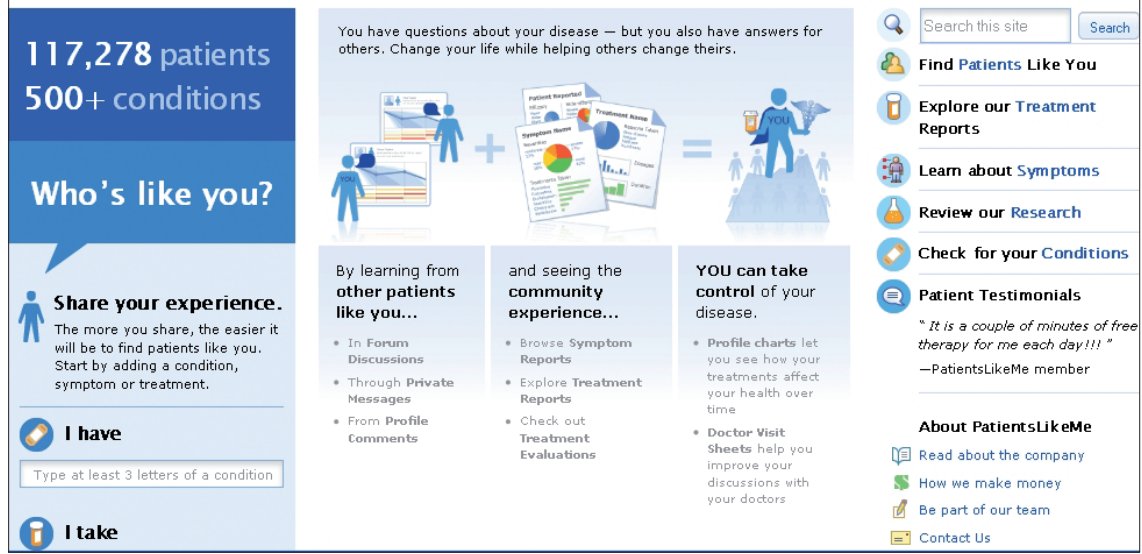

Patients like me, http://www.patientslikeme.com démica difunden una información muy precisa (Buis; Carpenter, 2009), por eso se les considera parte del grupo de medios de comunicación especializados en información sanitaria (Lagu et al., 2008). Sin embargo los blogs personales redactados por pacientes carecen en ocasiones de precisión informativa y de fiabilidad. De ahí la importancia de no otorgarles gran credibilidad en tanto que sus autores carecen de credenciales médicas y, por tanto, de autoridad científica. Son interesantes como vehículo para difundir experiencias médicas personales, pero no para otros fines como por ejemplo aconsejar a otros pacientes sobre el uso de medicamentos, o sobre el seguimiento de tratamientos médicos.

Las organizaciones sanitarias que apuestan por la creación de blogs pueden obtener varios beneficios. Ayudan a las organizaciones a comprender mejor las representaciones que se hacen los pacientes sobre la salud y la enfermedad (Legros, 2009), así como a promover la educación sanitaria del usuario, la prevención de enfermedades y la promoción de la salud (Adams, 2010a, p. e95). Constituyen un medio de comunicación institucional de salud. Por ello las organizaciones del sector deben considerar estas aplicaciones como parte integrante de sus estrategias de socialización.

Sin embargo, su utilización también comporta riesgos para las instituciones sanitarias:

- ponen de manifiesto el anonimato del paciente cuando se publican datos e imágenes suyas (Kamel-Boulos; Maramba; Wheeler, 2006);

- la falta de interacción social y de comunicación no verbal puede hacer que los mensajes tengan interpretaciones erróneas (Hillan, 2003);

- la reputación del profesional de la salud puede verse afectada por los comentarios difundidos en los blogs (Valverde, 2008).

Además de estos riesgos, hay que señalar el de la automedicación, es decir, la decisión del paciente de consumir fármacos según la información consultada en los blogs y no según lo indicado por un profesional de la salud. Esta situación supone un problema ya que según el estudio de la European Alliance for Access to Safe Medicines (2008), el 62\% de los medicamentos que se venden en internet son falsos o no cumplen con los estándares de calidad necesarios.

A pesar de los inconvenientes, y siguiendo con el citado protagonismo de las personas, cada vez son más comunes los blogs creados por y para pacientes, sin la participación de ningún organismo comercial. Cumplen una función social al hacer posible que los usuarios interesados por un mismo asunto médico se agrupen. Su importancia está en que, según indican Baker et al. (2003), la información de salud disponible en la Red puede ayudar al paciente a interactuar de manera más eficaz con el profesional de la salud cuando acude al hospital. En cualquier caso este protagonismo del usuario no puede relegar a un segundo plano el rol del médico, ya que gracias a este último puede tomar las decisiones acertadas y evitar las consecuencias negativas de una interpretación errónea de información sanitaria difundida en internet.

\section{Hay 11.751 blogs sobre salud (Technorati)}

\section{Tipos de blogs de salud}

El blog sanitario puede definirse según la autoría del contenido, como corporativo, personal, asociativo, mediático general, o mediático especializado.

El blog corporativo tiene una finalidad informativa, incluso educativa de la salud y en ocasiones comercial. El personal es sobre todo testimonial, mientras que el asociativo está enfocado hacia la colaboración abierta y la transmisión de experiencias terapéuticas. Por último, los blogs mediáticos se dirigen a grandes audiencias, y pueden versar sobre temas genéricos o especializados.

Las diferencias no siempre son evidentes y se entremezclan los modelos. En todos se puede decir que existe una orientación hacia la solución del problema clínico o médico, se produce una co-creación, y se realiza una actualización periódica de los contenidos.

Se pueden citar los siguientes ejemplos de cada modalidad: 
- Corporativos. Mayo Clinic y Harvard Medical School. http://sharing.mayoclinic.org

http://www.health.harvard.edu/blog

- Personales. Salud con cosas.

http://saludconcosas.blogspot.com

- Asociativos. Patients like me. http://www.patientslikeme.com

- Mediáticos generales. New York times, Washington post y Wall Street Journal.

http://well.blogs.nytimes.com

http://www.washingtonpost.com/blogs/the-checkup http://blogs.wsj.com/health

- Mediáticos especializados. Medicablogs de Diario médico y Jano.

http://medicablogs.diariomedico.com

http://www.jano.es/jano/ctl_servlet? $f=80$

Los equipos tecnológicos sobre los que se programan y diseñan favorecen la interacción y la socialización de los contenidos. Soluciones abiertas al público como Wordpress, Blogger o Typepad presentan los elementos mínimos para compartir contenido y enlazar con redes como Twitter o Facebook. Incluso es posible la creación de redes sociales autónomas en el blog mediante aplicaciones como Buddypress. Son innumerables los plug-ins y widgets diseñados para estos soportes que hacen posible el uso de calculadoras para el control de parámetros como el índice de masa corporal, el de dietas, o el de seguimiento de contenidos educativos como es el caso de Patient Education H1N1.

http://wordpress.org/extend/plugins/body-mass-indexcalculator-widget

http://wordpress.org/extend/plugins/protein-shake-recipecalculator

http://wordpress.org/extend/plugins/patient-educationh1n1-flu-tutorial

El blog de salud puede ser corporativo, personal, asociativo, o mediático

\section{Panorama mundial de los blogs de salud}

El número de blogs dedicados a la salud sigue creciendo de forma exponencial, principalmente los creados por y para pacientes. Según los datos aportados por Technorati -uno de los mejores motores de búsqueda especializados en blogs-, hay 11.751 blogs dedicados a la salud. Según el informe de Aitor Guitarte (2010), hay un total de 275 escritos en castellano, de los cuales la mayor parte tienen que ver con la atención primaria. En lo que se refiere a los blogs de salud más visitados, se pueden señalar los datos aportados por el Ranking Top Blogs Health de febrero de 2011, elaborado por Wikio, y según el cual los diez blogs más visitados son: 1) Well, 2) Kevin, M.D. -Medical weblog, 3) Respect ful insolence, 4) Science-based medicine, 5) The health care blog, 6) Pharmalot, 7) John Goodman's health policy blog, 8) In the pipeline, 9) Dr. Wes y 10) Age of autism.

http://technorati.com/blogs/directory/living/health http://www.wikio.com/blogs/top/health

El perfil del blogger de salud, siguiendo los datos aportados por Kovic, Lulic y Brumini (2008), se caracteriza porque tie- ne un nivel alto de educación, una edad entre 30 y 49 años, trabaja en la industria de la salud y vive en Estados Unidos. Las principales fuentes de información que utiliza son internet $(99 \%)$, blogs (86\%), newsletters (75\%), rss (really simple sindication) (63\%) y podcasts (24\%). Además, resulta interesante señalar que el $54 \%$ de los bloggers estudiados ha publicado alguna vez un artículo científico; el $44 \%$ un libro o un capítulo de libro; y el $41 \%$ un artículo de periódico, lo cual demuestra su alto nivel de educación.

El perfil profesional del blogger influye en el posicionamiento de su blog; es decir, la mayor parte de blogs se pueden clasificar o posicionar en función del área médica en que es experto el autor. Este criterio se aplica tanto a los creados por médicos como a los de pacientes, ya que, aunque estos últimos en ocasiones no tienen el mismo nivel de conocimiento que los profesionales, también crean y posicionan sus blogs en función de sus áreas de interés, sobre las cuales adquieren un cierto nivel de experiencia.

\section{Tendencias de futuro}

En el entorno sanitario, el uso de los blogs y el de las denominadas micro-redes o microblogging va enlazado y la integración entre ellos es ya una realidad. Es la denominada salud 2.0, la cual genera diversos comportamientos en la demanda de información y en el uso de los mismos medios sanitarios. Entre las tendencias de futuro merece la pena resaltar tres:

- demanda de información personalizada sobre las novedades médicas (Giustini, 2006), que implica tanto al profesional de la medicina como al público en general;

- promoción de la educación específica del personal sanitario y de la formación sanitaria general de la población. En este sentido se ha examinado la utilidad de los formatos en podcasts y la interacción con comunidades virtuales (Sandars; Haythornthwaite, 2007; Crespo, 2007);

- utilización de la Red por parte de facultativos y enfermeras para orientar su praxis y tomar así mejores decisiones (Hangwi, 2006).

Estas tendencias muestran la necesidad de que la información médica difundida en internet sea cada vez más fiable y precisa, lo cual repercutirá de modo positivo en las decisiones de salud adoptadas por el paciente $y$, consecuentemente, en su estado de salud.

Entre la población general, la variabilidad y la frecuencia de consulta de la información está en función del perfil del usuario y de la patología, lo que genera diferentes actitudes y usos de dichas fuentes en la Red (Ferguson, 2007). Hay que destacar la exposición o participación en investigaciones clínicas, fenómeno que se produce también en el entorno de la salud 2.0 (Frost et al., 2008).

Basándonos en las ideas defendidas por Ramaswamy (2010), de la University of Michigan, en los próximos años podemos atisbar un escenario caracterizado por un lado por el desarrollo de los entornos co-creativos, y por otro por el crecimiento no sólo de usuarios, sino también de contenidos emergentes mejor elaborados gracias al uso de los blogs. El que acceda al blog aportará un valor añadido cada vez mayor al profesional de la salud y a la misma comuni- 


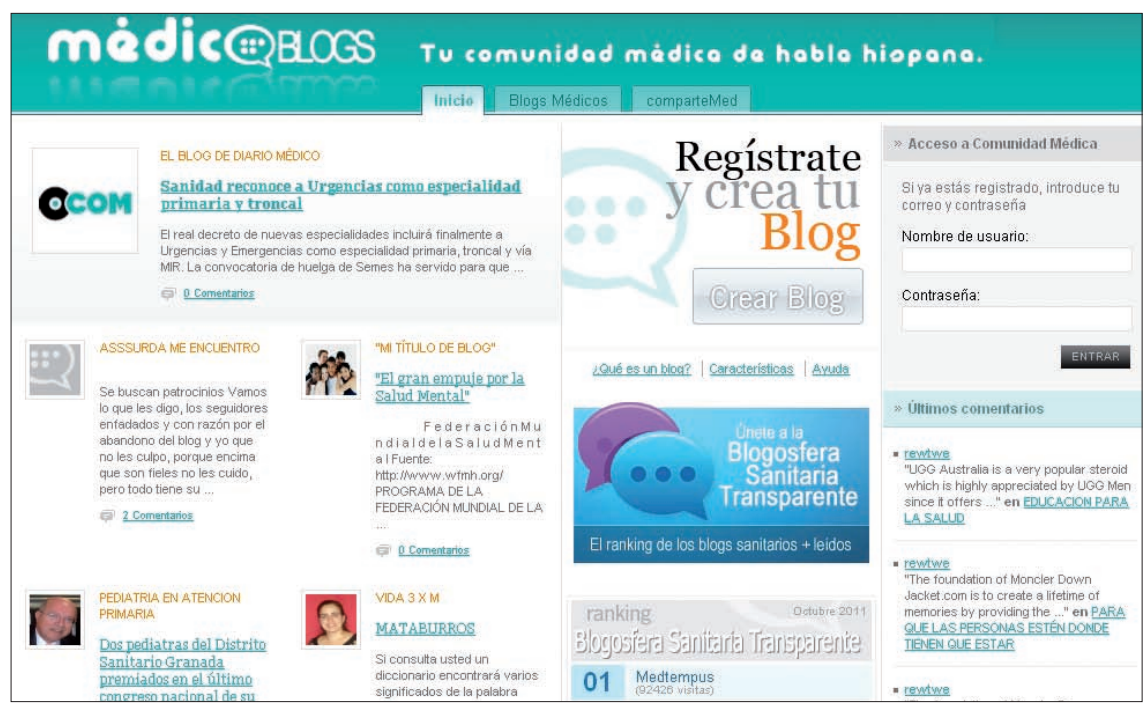

Medicablogs, http://medicablogs.diariomedico.com

dad de pacientes ya que al manejar información de mayor calidad y mejor elaborada, su contribución a la generación de un conocimiento fiable y preciso será cada vez mayor. Esto implica que es necesario garantizar mejor las responsabilidades de todos los implicados, tanto las legales como las relativas a los servicios recibidos.

La web 2.0 tiene un papel clave en la formación específica del profesional de la salud

El tono de la comunicación se mantendrá divergente entre los entornos corporativos y los personales o asociativos, dado que el primero protege la reputación de la propia marca o de la organización. El hospital quiere transmitir confianza y no admitirá la crítica o el comentario libre de un paciente o afectado. Los blogs mediáticos tienen por su parte un horizonte por explorar en cuanto a la colaboración más estrecha con asociaciones y grupos de autoayuda de los que se pueden enriquecer a nivel informativo, pero con los que también pueden colaborar mediante la provisión de datos y contactos de máxima importancia para los afectados.

En su formato, la integración es un proceso que ya está en marcha y el microblogging trae como consecuencia una reacción y una capacidad de respuesta mucho mayor y mejor organizada por parte de las comunidades de pacientes y de los profesionales de la medicina. Por otro lado, la adaptación de estos contenidos y funcionalidades a los dispositivos móviles mediante aplicaciones es un presente que ya se experimenta y que ayuda a potenciar la utilidad de los mismos. La población, ya sea como uso preventivo o terapéutico de la información, se comporta de un modo diferente, complementando el conocimiento obtenido en internet con el uso de equipos que le capacitan para incorporar o confirmar esos datos mediante aplicaciones móviles. Hay más de un centenar de aplicaciones relacionadas con la salud y con la calidad de vida para iPhone o iPad que pueden encontrarse en la Red y que facilitan cálculos y mediciones hasta ahora desconocidos para la mayoría de los ciudadanos. http://www.uspharmd.com/blog/ 2009/100-fabulous-iphone-apps-foryour-health-and-fitness

Ante dicho cambio tanto los centros sanitarios como los grupos de autoayuda o colectivos de pacientes necesitarán una estrategia adecuada para aprovechar el potencial y buscar metas concretas para la mejora de la salud mediante las nuevas redes. En poco tiempo los hospitales y los sanitarios verán pacientes mejor informados, o desorientados, según la información recibida. Tendrán soluciones y se habrán organizado antes de que la misma administración sanitaria o el colectivo profesional hayan buscado una solución a sus dificultades. Pero sea cual sea el cambio, tanto unos como otros se necesitan, y para ello los actuales dispositivos como el blog o el microblogging juegan un papel esencial para poder avanzar juntos.

El riesgo es entender este cambio exclusivamente como una oportunidad para lograr ahorros de costes sanitarios, cuando realmente lo que debe perseguir es la mejora de la calidad asistencial y de la eficacia terapéutica, para lo cual, la difusión de información médica de calidad resulta fundamental. En cualquier caso, ambas opciones pueden ser compatibles ya que al fin y al cabo la web 2.0 constituye un nuevo punto de encuentro entre el profesional de la salud y el paciente que influye positivamente en el servicio de salud ofrecido por los hospitales.

\section{Bibliografía}

Adams, Samantha. "Blog-based applications and health information: two case studies that illustrate important questions for consumer health informatics (CHI) research". International journal of medical informatics, 2010a, v. 79, n. 6, pp. e89-e96.

http://dx.doi.org/10.1016/j.ijmedinf.2008.06.009

Adams, Samantha. "Revisiting the online health information reliability debate in the wake of "web 2.0": an interdisciplinary literature and website review". International journal of medical informatics, 2010b, v. 79, n. 6, pp. 391-400.

http://dx.doi.org/10.1016/j.ijmedinf.2010.01.006

Baker, Laurence; Wagner, Todd; Singer, Sara; Bundorf, Kate. "Use of the internet and email for health care information: results from a national survey". Journal of the American Medical Association, 2003, v. 289, n. 18, pp. 2400-2406. http://dx.doi.org/10.1001/jama.289.18.2400

Bennett, Gary; Glasgow, Russell. "The delivery of public health interventions via the internet: actualizing their potential". Annual review of public health, 2009, v. 30, pp. 273292.

http://dx.doi.org/10.1146/annurev.publhealth.031308. 100235

Buis, Lorraine; Carpenter, Serena. "Health and medical blog content and its relationships with blogger credentials and 
blog host". Health communication, 2009, v. 24, n. 8, pp. 703 710.

http://dx.doi.org/10.1080/10410230903264014

Comscore. Online health information category grows at rate four times faster than total internet. 9 september 2008. http://www.comscore.com/Press_Events/Press_Releases/ 2008/09/Top_Internet_Healths_Sites/(language)/eng-US

Crespo, Richard. "Virtual community health promotion". Preventing chronic disease, 2007, v. 4, n. 3, pp. 75. http://www.ncbi.nlm.nih.gov/pmc/articles/PMC1955425

Denecke, Kerstin. "Assessing content diversity in medical weblogs?" En: First International Workshop on Living Web: making web diversity a true asset held in conjunction with the International Semantic Web Conference, Fairfax, United States, 25 October 2009.

http://livingknowledge.europarchive.org/images/publica tions/LivingWeb.pdf

European Alliance for Access to Safe Medicines. The counterfeiting superhighway: the growing threat of online pharmacies. Cardiff, UK: European Alliance for Access to Safe Medicines, 2008.

http://v35.pixelcms.com/ams/assets/312296678531/455_ EAASM_counterfeiting\%20report_020608.pdf

Eysenbach, Gunther. "Medicine 2.0: social networking, collaboration, participation, apomediation, and openness". Journal of medical internet research, 2008, v. 10, n. 3, pp. e22. http://dx.doi.org/10.2196/jmir.1030

Ferguson, Tom. E-patients: how they can help us heal health care, 2007.

http://www.e-patients.net/e-Patients_White_Paper.pdf

Fostier, Pierrik. "Les patients de culture différente". En: Richard, Claude; Lussier, Marie-Thérèse (eds.).La communication professionnelle en santé. Québec: ERPI, Éditions du Renouveau Pédagogique Inc., 2005, pp. 461-482.

Frost, Jeana; Massagli, Michael; Wicks, Paul; Heywood, James. "How the social web supports patient experimentation with a new therapy: the demand for patient-controlled and patient-centered informatics". AMIA Annual Symposium Proceedings, 2008, pp. 217-221.

http://www.ncbi.nlm.nih.gov/pmc/articles/PMC2656086

Fundación Telefónica. La sociedad de la información en España 2010. Madrid: Fundación Telefónica, 2010.

http://e-libros.fundacion.telefonica.com/sie10/aplicacion_ sie/partea/datos.html

Giustini, Dean. "How web 2.0 is changing medicine". British medical journal, 2006, v. 333, n. 7582, pp. 1283-1284.

http://www.bmj.com/content/333/7582/1283.full

http://dx.doi.org/10.1136/bmj.39062.555405.80

Guitarte, Aitor. "Blogs sanitarios en español. Activos y ordenados". Somosmedicina.com, febrero 2010.

http://www.somosmedicina.com/2010/02/blogs-sanitariosen-espanol-activos-y_24.html

Hillan, Julie. "Physician use of patient-centered weblogs and online journals". Clinical medicine \& research, 2003, v. 1, n. 4, pp. 333-335.
http://www.ncbi.nlm.nih.gov/pmc/articles/PMC1069063

Kamel-Boulos, Maged; Maramba, Inocencio; Wheeler, Steve. "Wikis, blogs and podcasts: a new generation of webbased tools for virtual collaborative clinical practice and education". BMC medical education, 2006, v. 6, p. 41. http://www.ncbi.nlm.nih.gov/pmc/articles/PMC1564136 http://dx.doi.org/10.1186/1472-6920-6-41

Kamel-Boulos, Maged; Wheeler, Steve. "The emerging web 2.0 social software: an enabling suite of sociable technologies in health and health care education". Health information and libraries journal, 2007, v. 24, n. 1, pp. 2-23.

Kovic, Ivor; Lulic, Ileana; Brumini, Gordana. "Examining the medical blogosphere: an online survey of medical bloggers". Journal of medical internet research, 2008, v. 10, n. 3, pp. e28.

http://www.jmir.org/2008/3/e28/

http://dx.doi.org/10.2196/jmir.1118

Lagu, Tara; Kaufman, Elinore; Asch, David; Armstrong, Katrina. "Content of weblogs written by health professionals". Journal of general internal medicine, 2008, v. 23, n. 10, pp. 1642-1646.

http://www.pharmalot.com/wp-content/uploads/2008/07/ medical-blogs.pdf

Legros, Michel. "Étude exploratoire sur les blogs personnels santé et maladie". Santé publique, 2009, hs2, v. 21, pp. 4151.

http://www.cairn.info/revue-sante-publique-2009-hs2page-41.htm

Orihuela, José-Luis. La revolución de los blogs: cuando las bitácoras se convirtieron en el medio de comunicación de la gente. Madrid: La esfera de los libros, 2006, ISBN: 8497344987.

Parrada, Antoni. "Portales sanitarios en España". El profesional de la información, 2000, v. 9, n. 12, pp. 26-31.

http://www.elprofesionaldelainformacion.com/conteni dos/2000/diciembre/4.pdf

Pfizer. ¿Hasta dónde llega la responsabilidad de los pacientes y de los médicos? Madrid: Pfizer, 2010.

Rius, Roser; González, José-Antonio. "Software libre: una oportunidad para los investigadores". Medicina clínica, 2011 , v. 136 , n. 5 , pp. 205-206.

Ramaswamy, Venkat. "Competing through co-creation: innovation at two companies". Strategy \& leadership, 2010, v. 38, n. 2, pp. 22-29.

http://www.emeraldinsight.com/journals. htm ?articleid $=18$ 45912\&show $=$ html

http://dx.doi.org/10.1108/10878571011029028

Sánchez-Navarro, Jordi; Aranda, Daniel. "Internet como fuente de información para la vida cotidiana de los jóvenes españoles". El profesional de la información, 2011, v. 20, n. 1, pp. 32-37.

http://eprints.rclis.org/bitstream/10760/15332/1/032-037.pdf http://dx.doi.org/10.3145/epi.2011.ene.04

Sandars, John; Haythornthwaite, Caroline. "New horizons for e-learning in medical education: ecological and web 2.0 
